\begin{tabular}{|c|l|}
\hline Title & Mean curvature flow closes open ends of noncompact surfaces of rotation \\
\hline Author(s) & Giga, Y oshikazu; Seki, Y ukihiro; Umeda, Noriaki \\
\hline Citation & Hokkaido University Preprint Series in Mathematics, 938, 1-22 \\
\hline Issue Date & 2009-2-20 \\
\hline DOI & 10.14943/84086 \\
\hline Doc URL & http://hdl.handle.net/2115/69746 \\
\hline Type & bulletin (article) \\
\hline File Information & pre938.pdf \\
\hline
\end{tabular}

Instructions for use 


\title{
Mean curvature flow closes open ends of noncompact surfaces of rotation
}

\author{
Yoshikazu Giga, Yukihiro Seki ${ }^{1}$ and Noriaki Umeda \\ Graduate School of Mathematical Sciences, University of Tokyo, \\ 3-8-1 Komaba, Meguro-ku Tokyo 153-8914, Japan
}

\begin{abstract}
We discuss the motion of noncompact axisymmetric hypersurfaces $\Gamma_{t}$ evolved by mean curvature flow. Our study provides a class of hypersurfaces that share the same quenching time with that of the shrinking cylinder evolved by the flow and prove that they tend to a smooth hypersurface having no pinching neck and having closed ends at infinity of the axis of rotation as the quenching time is approached. Moreover, they are completely characterized by a condition on initial hypersurface.
\end{abstract}

Keywords: axisymmetric hypersurface; mean curvature flow; quenching.

AMS Subject Classification: 53C44; 35K55; 35K15.

\section{Introduction and main results}

We discuss the motion of noncompact hypersurfaces $\Gamma_{t}$ evolved by mean curvature flow

$$
V=H \quad \text { on } \Gamma_{t}
$$

where $V$ and $H$ denote normal velocity and mean curvature of the hypersurfaces, respectively. (Here and henceforth we do not take average of principal curvatures to define mean curvature.) We assume that initial hypersurface $\Gamma_{0}$ is rotationally symmetric to an axis and is represented by rotating the graph of a positive function $u_{0}$ around the axis. Then $\Gamma_{t}$ remain rotationally symmetric to the axis so long as they exist (cf. [1, Theorem 4.3a]). Namely, we may assume that the hypersurfaces are given of the form

$$
\Gamma_{t}=\left\{X=\left(x, y_{1}, \ldots, y_{n}\right) \in \mathbf{R}^{n+1} \mid r=u(x, t)\right\}
$$

with some function $u$, where $r=\left(\sum_{j=1}^{n} y_{j}^{2}\right)^{1 / 2}$ denotes the distance from the $x$-axis to $\Gamma_{t}$. We are interested in the behavior of $\Gamma_{t}$ evolved by (1.1) when the initial hypersurface has two open ends at the space infinity. Our goal is to give a condition that an open ends close in finite time with no neck-pinch.

\footnotetext{
${ }^{1}$ Corresponding to: Yukihiro Seki, Graduate School of Mathematical Sciences, University of Tokyo, Tokyo, 153-8914, Japan. E-mail: seki@ms.u-tokyo.ac.jp
} 
Mean curvature flow equation (1.1) is rephrased by $u$ as

$$
u_{t}=\frac{u_{x x}}{1+u_{x}^{2}}-\frac{n-1}{u}, \quad x \in \mathbf{R}, t>0
$$

(cf. $\S 2$ ). We now consider an initial value problem for a noncompact surface of rotation with two open ends satisfying (1.1). This problem can be formulated by the Cauchy problem for (1.3) with initial condition

$$
u(x, 0)=u_{0}(x)>0, \quad x \in \mathbf{R} .
$$

Throughout the present article, we assume

$$
\begin{aligned}
& u_{0} \text { is bounded and uniformly continuous in } \mathbf{R} ; \\
& m:=\inf _{x \in \mathbf{R}} u_{0}(x)>0 .
\end{aligned}
$$

The Cauchy problem (1.3)-(1.4) has a unique positive classical solution locally in time under the assumption $(H 1)$ and $(H 2)$. (cf. $\S 2$. We do not need notion of viscosity solution due to $[4,8]$ except for $\S 5$.) We remark that the solution is forced to reach zero in finite time, referred as quenching in finite time, as long as bounded initial data are concerned. This fact is readily seen if one compares the solution with a spatially homogeneous solution $v_{M}(t)=$ $\sqrt{2(n-1)(T(M)-t)}$, where $M=\sup _{x \in \mathbf{R}} u_{0}(x)$ and $T(M)=M^{2} / 2(n-1)$. Once a solution reaches zero, the equation (1.3) does not make sense and hence the solution cannot be extended globally in time as a classical solution. For a given initial datum $u_{0}$, we set

$$
T\left(u_{0}\right)=\sup \left\{t>0 ; \inf _{x \in \mathbf{R}} u(x, t)>0\right\}<\infty
$$

and call it the quenching time of $u$. It is immediate that

$$
\liminf _{t \nearrow T\left(u_{0}\right)} \inf _{x \in \mathbf{R}} u(x, t)=0 .
$$

We are concerned with the quenching time and with the behavior of solution $u$ when the quenching time is approached.

A point $a \in \mathbf{R}$ is said to be a quenching point (or pinching point) of $u$ if there exists a sequence $\left\{\left(x_{k}, t_{k}\right)\right\} \subset \mathbf{R} \times\left(0, T\left(u_{0}\right)\right)$ such that

$$
x_{k} \rightarrow a, t_{k} \nearrow T\left(u_{0}\right) \text { and } u\left(x_{k}, t_{k}\right) \rightarrow 0 \quad \text { as } k \rightarrow \infty .
$$

In other words, quenching points of $u$ correspond to positions of pinching necks of the hypersurface $\Gamma_{t}$ at $t=T\left(u_{0}\right)$. The simplest example is a family of cylinders associated with constant initial function $u_{0} \equiv m>0$. In this case, the solution of (1.3)-(1.4) coincides with the solution $v_{m}(t)$ of the ordinary differential equation

$$
v^{\prime}=-\frac{n-1}{v}, t>0 ; \quad v(0)=m,
$$

that is,

$$
v_{m}(t)=\sqrt{2(n-1)(T(m)-t)} \quad \text { with } \quad T(m)=\frac{m^{2}}{2(n-1)} .
$$


The set of quenching points of $v_{m}$ is nothing but the whole real line $\mathbf{R}$, and the corresponding hypersurfaces are cylinders $\left\{r=v_{m}(t)\right\}$ shrinking monotonically toward to the axis and vanishing at the time $T(m)$.

There is a large number of literature on formation of singularities of the mean curvature flow for compact hypersurfaces. However, as far as the authors know, there seem to be no articles discussing singularities at space infinity of noncompact hypersurfaces.

For a compact hypersurface in $\mathbf{R}^{n+1}$ convexity is preserved and the hypersurface shrinks asymptotically self-similarly to a point ([22] for $n \geq 2$ ) ([11] for $n=1)$ without developing singularities. It shrinks asymptotically like a sphere. For a curve evolution even if the initial (embedded) curve is non-convex the solution becomes convex without developing singularities and self-intersections [20]. However, for higher dimensional case if the initial hypersurface is nonconvex, the solution may develop singularities in finite time as pointed out by [21] by giving an example of a thin-neck barbell-like surface: two spherical surface connected by a thin "neck". Inward curvature of the neck is so large that it forces the neck to pinch before the two spherical parts shrink.

In general (not necessarily for axisymentric hypersurfaces) it is known that the Hausdorff dimension of the singularity is less than or equal to $n-1$ by $[37,38]$ provided that the initial hypersurface is mean-convex. (This is optimal since a regular doughnut (solid torus) shrinks to a ring.) About formation of singularities the reader is referred to a recent book of [7] and papers cited there.

There are several methods to extend a solution after it develops singularities. The first one is the varifold solution introduced by [3]. The theory is developed by $[24,34,36]$, where the varifold solution is constructed as a limit of the diffused interval layer of the Allen-Cahn equation. Another one is a level set solution introduced by $[4,8]$ based on the theory of viscosity solutions [5]. The reader is referred to [12] and [13] and references cited there on the level set approach.

The way of shrinking for axisymmetric barbell has been studied. It turns out that neck-pinching is isolated. This is first observed by [6] under several technical assumptions like symmetry $u(x, t)=u(-x, t)$. Moreover, the singularity is type $I$ in the sense the rate of blowup of curvature is self-similar like $(T-t)^{-1 / 2}$ where $T$ is the quenching (pinching) time ([35] for special hypersurface and [1] for general hypersurfaces.) The reader is referred to a recent book of M.-H. Giga and Y. Giga and J. Saal [14, Chapter 3] for its background. Moreover, type II singularity where the blowup of curvature is faster than self-similar rate are constucted in [1] and more explicity by [2] by constructing 'degenerate pinching'. See also [12])

As for general axisymmetric (compact) hypersurfaces, Altschuler, Angenent and Giga [1] prove that there exists a finite sequence $0=t_{0}<t_{1}<\ldots<t_{\ell}$ such that hypersurfaces $\Gamma_{t}$ are smooth and compact in all of $\mathbf{R}^{n+1}$ for $t_{j-1}<t<t_{j}$, $j=1,2, \ldots, \ell$, and the number of components can change only at singular times $t_{j}(j=1,2, \ldots, \ell)$. The solution is empty for $t>t_{\ell}$. They also prove that, if $\Gamma_{*}(t)$ represents a component of $\Gamma_{t}$ that becomes singular at the time $t_{j}$, the following alternative holds: (i) $\Gamma_{*}(t)$ shrinks to a point on the axis of rotation; (ii) one or one more "necks" have been pinched. Notice, however, that we do not know if each component of the hypersurfaces actually pinches on the axis at the singular times $t_{j}$.

Our aim consists in showing the existence of hypersurfaces having no pinching neck on the axis at a minimal quenching time, a notion defined below, and 
in characterizing such hypersurfaces by a condition on initial hypersurface.

Among the solutions of the Cauchy problem (1.3)-(1.4), we are interested in the ones that share the same quenching time with $v_{m}(t)$ in (1.6). We say that such a solution has a minimal quenching time.

Definition 1.1. A solution $u$ of the Cauchy problem (1.3)-(1.4) is said to have a minimal quenching time, if

$$
T\left(u_{0}\right)=T(m) .
$$

As is proved in $\S 4$, a necessary condition for a solution $u$ of (1.3)-(1.4) to have a minimal quenching time is that

$$
\liminf _{x \rightarrow-\infty} u(x, t)=v_{m}(t) \quad \text { or } \quad \liminf _{x \rightarrow+\infty} u(x, t)=v_{m}(t)
$$

for every $t \in[0, T(m))$. Therefore a typical hypothesis on initial function is

$$
\lim _{x \rightarrow-\infty} u_{0}(x)=m
$$

or

$$
\lim _{x \rightarrow+\infty} u_{0}(x)=m,
$$

where $m>0$ is the infimum of $u_{0}$ as is stated in (H2). We consider the following conditions, which are weaker than (1.7) or (1.8):

There exists a sequence $\left\{x_{k}\right\} \subset \boldsymbol{R}$ such that $x_{k} \rightarrow \infty$ and

$$
u_{0}\left(x+x_{k}\right) \rightarrow m \quad \text { a.e. as } k \rightarrow \infty \text {; }
$$

There exists a sequence $\left\{x_{k}\right\} \subset \boldsymbol{R}$ such that $x_{k} \rightarrow-\infty$ and

$$
u_{0}\left(x+x_{k}\right) \rightarrow m \quad \text { a.e. as } k \rightarrow \infty \text {. }
$$

In face, the above convergence holds at each point $x \in \mathbf{R}$ since we assume uniform continuity for $u_{0}$. Under these assumptions, we are able to prove that a solution of (1.3)-(1.4) has a minimal quenching time and quenches at spatial infinity. Here a solution $u$ of (1.3)-(1.4) with quenching time $T\left(u_{0}\right)$ is said to quench at spatial infinity if there exists a sequence $\left\{\left(x_{n}, t_{n}\right)\right\} \subset \mathbf{R} \times\left(0, T\left(u_{0}\right)\right)$ such that

$$
\left|x_{n}\right| \rightarrow \infty, t_{n} \nearrow T\left(u_{0}\right) \text { and } u\left(x_{n}, t_{n}\right) \rightarrow 0 \quad \text { as } n \rightarrow \infty .
$$

Theorem 1.2. Assume (H1) and (H2). If initial data $u_{0}$ satisfies condition (1.9) (or (1.10)), then a solution $u$ of the Cauchy problem (1.3)-(1.4) has a minimal quenching time. Moreover, for any $\varepsilon, T \in(0, T(m))$ and $R>0$,

$$
\lim _{k \rightarrow \infty} \sup _{|x| \leq R, \varepsilon \leq t \leq T}\left|u\left(x+x_{k}, t\right)-v_{m}(t)\right|=0,
$$

where the sequence $\left\{x_{k}\right\}$ is as in condition (1.9) (or (1.10)), and the solution quenches at spatial infinity. Furthermore, if the hypothesis (1.7) (or (1.8)) is true, then

$$
\lim _{x \rightarrow \infty} u(x, t)=v_{m}(t) \quad\left(\text { or } \quad \lim _{x \rightarrow-\infty} u(x, t)=v_{m}(t)\right)
$$

and the convergence is uniform on compact subsets of $(0, T(m))$. 
Theorem 1.2 states that (1.9) (or (1.10)) is a sufficient condition for a solution of (1.3)-(1.4) to have a minimal quenching time. In the last part of this introduction, it is shown in fact to be a necessary and sufficient condition (Theorem 1.4). As for such a solution, we are able to prove that the solution is positive everywhere in $\mathbf{R}$ up to the quenching time unless it starts from a flat datum. As a result, we get its smooth limit function as the quenching time is approached. In other words, the corresponding hypersurfaces $\Gamma_{t}$ have no pinching neck at the quenching time and tend to a smooth limit hypersurface.

Theorem 1.3. Assume $(H 1)$ and $(H 2)$. Let $u$ be a solution of the Cauchy problem (1.3)-(1.4) having a minimal quenching time $T(m)$. If $u_{0} \not \equiv m$, then there is no quenching point of $u$. Moreover, there exists a function $u(\cdot, T(m)) \in$ $C^{\infty}(\boldsymbol{R})$ such that $u(\cdot, t) \rightarrow u(\cdot, T(m))$ in the Frechét space $C^{\infty}(\boldsymbol{R})$ as $t \nearrow T(m)$, $u(x, T(m))>0$ in the whole $\boldsymbol{R}$ and

$$
\liminf _{x \rightarrow-\infty} u(x, T(m))=0 \text { or } \liminf _{x \rightarrow+\infty} u(x, T(m))=0 .
$$

The latter statement is a direct consequence of nonexistence of quenching points and standard parabolic estimates. It tells us the presence of smooth limit surface which shrinks at spatial infinity.

Theorem 1.3 may remaind the reader of a version of the strong maximum principle established in Giga, Ohnuma and Sato [15] for the level set minimal surface equation. They investigate the large time behavior of viscosity solutions of the level set equation for the generalized mean curvature flow with the (homogeneous) Neumann boundary condition in a cylindrical domain $\Omega$ of the form

$$
\Omega=\left\{x=\left(x^{\prime}, x_{n}\right) \in \mathbf{R}^{n} ; x^{\prime} \in \Omega^{\prime}, x_{n} \in \mathbf{R}\right\},
$$

where $\Omega^{\prime}$ is a smoothly bounded domain in $\mathbf{R}^{n-1}$ with $n \geq 2$. Among other things, they showed that if initial data is constant in which $\left|x_{n}\right|$ is large enough, then a solution converges uniformly on $\bar{\Omega}$ as $t \rightarrow \infty$ to a solution of the level set minimal surface equation with the Neumann boundary condition. On the other hand, we are mainly concerned with the behavior of a classical solution of (classical) mean curvature flow equation (1.1) up to its quenching time. Our result described in Theorem 1.3 guarantees that if an initial hypersurface is different from a cylinder, then a solution never agrees with any cylinder up to its quenching time provided that the quenching time is minimal.

As is previously noticed, we obtain a necessary and sufficient condition on initial data for a solution of the Cauchy problem (1.3)-(1.4) to have a minimal quenching time as a consequence of Theorems 1.2 and 1.3.

Theorem 1.4. Assume $(H 1)$ and $(H 2)$. Then a solution of the Cauchy problem (1.3)-(1.4) has a minimal quenching time if and only if the initial datum satisfies conditions (1.9) or (1.10). Moreover, the limit function obtained in Theorem 1.3 satisfies for any $R>0$,

$$
\lim _{k \rightarrow \infty} u\left(x+x_{k}, T(m)\right)=0 \quad \text { if }|x| \leq R,
$$

where $\left\{x_{k}\right\}$ is the sequence as in (1.9) (or (1.10)). Furthermore, if the hypothesis (1.7) (or (1.8)) is true, then

$$
\lim _{x \rightarrow \infty} u(x, T(m))=0 \quad\left(\text { or } \lim _{x \rightarrow-\infty} u(x, T(m))=0\right) .
$$


Requiring in the proof of Theorem 1.4, we introduce equivalent conditions of (1.9) and (1.10) by a weighted mean value of initial data defined by

$$
A_{\rho}\left(x ; u_{0}\right)=\left(\rho * u_{0}\right)(x)=\int_{-\infty}^{\infty} \rho(x-y) u_{0}(y) d y
$$

with $\rho \in L^{1}(\mathbf{R})$ being a positive smooth function such that $\|\rho\|_{1}=1$. For example, we may choose $\rho(x)=\pi^{-1 / 2} \exp \left(-|x|^{2}\right)$.

Remark 1.5. Conditions (1.9) and (1.10) are equivalent respectively to conditions (1.17) and (1.18) below:

$$
\begin{aligned}
& \text { There exists a sequence }\left\{x_{k}\right\} \subset \boldsymbol{R} \text { such that } x_{k} \rightarrow \infty \text { and } \\
& \qquad A_{\rho}\left(x_{k} ; u_{0}\right) \rightarrow m \text { as } k \rightarrow \infty
\end{aligned}
$$

There exists a sequence $\left\{x_{k}\right\} \subset \boldsymbol{R}$ such that $x_{k} \rightarrow-\infty$ and

$$
A_{\rho}\left(x_{k} ; u_{0}\right) \rightarrow m \text { as } k \rightarrow \infty .
$$

The equivalence of these conditions may be proved in the same way as in [32, Appendix B], where a similar equivalence on initial data is proved, and thereby the proof of the equivalence above is safely left for the readers.

Theorems 1.2 and 1.3 is an analogue of the recent results of $[17,18,32,30]$ for related blow-up problems. Giga and Umeda [17, 18] studied semilinear equations $u_{t}=\Delta u+f(u)$ with $f(u)=u^{p}, p>1$, being a typical example of nonlinear term, and proved that blow-up occurs only at space infinity if initial datum is not a constant and takes its maximum at infinity. Their results were generalized in $[32,30]$ for degenerate quasilinear parabolic equations

$$
u_{t}=\Delta \phi(u)+f(u)
$$

where typical examples of nonlinear terms are $\phi(u)=u^{m}$ and $f(u)=u^{p}$ with $m>0$ and $p>1$. The notion of the least (possible) blow-up time (or minimal blow-up time) was originally introduced in [32] and the authors of [32] revealed the fact that the solutions having such a blow-up time may be characterized by initial data, which had been hidden in the results of [18]. That notion is closely related to the notion of minimal quenching time given in Definition 1.1 of the present article. Theorem 1.4 is a variant of those results. On the other hand, equation (1.1) admits a solution extending as a level set solution (introduced by $[4,8]$ based on the theory of viscosity solution; see e.g. [5]) after quenching time. It contrasts with the corresponding result for (1.19) with $\phi(u)=u^{m}$, $m \geq 1$, where every solution with minimal blow-up time exhibits "complete blow-up", that is, it has no reasonable extension after the blow-up time [33, 31]. Concerning blow-up at space infinity, the reader is referred to $[26,19]$ for earlier results, [16] for survey and [33] for recent development on the blow-up profile.

The reader may be interested in the behavior of solution after quenching time. It is known that there is a global-in-time level set solution as discussed in [1]. The results in [1] states for compact hypersurfaces; however, it can be extended for noncompact hypersurfaces [13]. In this paper we just remark that our level set solution becomes compact instantaneously after the quenching time under the assumption (1.7) (or (1.8)). We do not focus the problem whether the generalized solution develops fattening, which is not expected in our setting. 
The strategies to prove the theorems are the same in spirit with those of $[32,30]$. Indeed, if the term $u_{x x} /\left(1+u_{x}^{2}\right)$ in equation (1.3) were replaced by $u_{x x}$, then the same argument with $[32,30]$ works for our problem after a certain transformation of the arguments and thereby analogous results hold for Kawarada's equation [25]

$$
u_{t}=u_{x x}+\frac{1}{1-u} .
$$

However, the quasilinear structure of equations (1.19) used in [32, 30] is somewhat different from ours. The form of the diffusion term $\Delta \phi(u)$ in (1.19) is convenient for weak formulation of solutions based on integration by parts, but it is difficult to rewrite the right hand side of equation (1.3) in such a form. Accordingly, a different approach must be required. We use a gradient estimate due to [1] to control the term $u_{x x} /\left(1+u_{x}^{2}\right)$ in order to prove Theorem 1.2. Our basic approach to Theorem 1.3 is to construct a suitable subsolution in each bounded spatial interval. Single-point pinching lemma, which is recalled in $\S 2$ together with the gradient estimate, plays a crucial role in the construction. Tools from the theory of linear parabolic equations, such as fundamental solutions, the strong maximum principle and various parabolic estimates, are repeatedly used throughout the article.

The rest of the article is organized as follows. In the next $\S 2$ we briefly summarize some fundamental results such as local well-posedness of the Cauchy problem and recall basic tools from [1]. In $\S 3$ we implement the comparison argument mentioned above to prove Theorem 1.3. Theorem 1.2 is a direct consequence of Proposition 4.1 proved in the former part of $\S 4$. The latter part of $\S 4$ is devoted to proving the necessary and sufficient condition for minimal quenching time described in Theorem 1.4. Finally profile after quenching is shortly discussed in $\S 5$.

\section{Local well-posedness and preliminaries}

In this section we summarize some fundamental facts concerning existence and uniqueness of a local-in-time classical solution of the Cauchy problem (1.3)-(1.4) and recall some basic results from [1].

Although the equation (1.3) was already derived in [21,9] for $n=2$ and in [1] for general $n$ by the radial distance $u$ of the hypersurfaces to the axis of rotation, we give another way to derive the equation using the level set method (cf. [13]), which describes a hypersurface as the zero level set of an auxiliary function. Under the assumption (1.2), we may select an auxiliary function as

$$
\phi(x, t):=-r+u(x, t)
$$

so that the hypersurfaces are represented as $\Gamma_{t}=\left\{X \in \mathbf{R}^{n+1} \mid \phi(X, t)=0\right\}$. Here we observe that $|\nabla \phi|=\left(1+u_{x}^{2}\right)^{1 / 2}$ does not vanish on $\Gamma_{t}$. With this function, we may choose a unit normal vector field $\mathbf{n}$ of $\Gamma_{t}$ by $\mathbf{n}=-\nabla \phi /|\nabla \phi|$, so that $V$ and $H$ are

$$
\begin{aligned}
& V=\frac{\phi_{t}}{|\nabla \phi|}=\frac{u_{t}}{\left(1+u_{x}^{2}\right)^{1 / 2}}, \\
& H=-\nabla \cdot \mathbf{n}=\nabla \cdot\left(\frac{\nabla \phi}{|\nabla \phi|}\right)=\frac{u_{x x}}{\left(1+u_{x}^{2}\right)^{3 / 2}}-\frac{1}{\left(1+u_{x}^{2}\right)^{1 / 2}} \frac{n-1}{r},
\end{aligned}
$$


We begin with proving the existence of smooth solution of (1.3)-(1.4) with bounded uniformly continuous initial data. Such a result is essentially known based on a priori estimates [27]. However, we rather give its proof based on analytic semigroup theory [28, 29] for completeness and readers' convenience, since it is a nice application of the theory.

In order to prove such a result, we first show that local-in-time existence and uniqueness of solution to the Cauchy problem for smooth data are obtained via the theory of analytic semigroup developed in [28] for abstract quasilinear evolution equations. Let $\mathcal{X}$ be a Banach space and let $D$ be a subspace of $\mathcal{X}$. For $0<\theta<1$, we denote by $(\mathcal{X}, D)_{\theta, \infty}$ a real interpolation space between $\mathcal{X}$ and $D$. For fixed $T>0$ and $\theta \in(0,1)$, let $U$ be an open set in $(\mathcal{X}, D)_{\theta, \infty}$ and suppose that the mappings:

$$
\begin{aligned}
& A(t, u):[0, T] \times U \rightarrow L(D, \mathcal{X}), \\
& f(t, u):[0, T] \times U \rightarrow \mathcal{X},
\end{aligned}
$$

are Hölder continuous with exponent $\gamma \in(0,1)$ with respect to $t$, and locally Lipschitz continuous with respect to $u$, i.e.:

(i) For any $u_{0} \in U$, there exist constants $K>0$ and $r>0$ such that

$$
\|A(t, u)-A(s, v)\|_{L(D, \mathcal{X})}+\|f(t, u)-f(s, v)\|_{\mathcal{X}} \leq K\left(|t-s|^{\gamma}+\|u-v\|_{\theta, \infty}\right)
$$

for all $u, v$ in a closed ball $B\left(u_{0}, r\right) \subset(\mathcal{X}, D)_{\theta, \infty}$.

Assume moreover:

(ii) For any $t_{0} \in[0, T]$ and $u_{0} \in U$, the operator $A\left(t_{0}, u_{0}\right): D\left(A\left(t_{0}, u_{0}\right)\right) \subset$ $\mathcal{X} \rightarrow \mathcal{X}$ is a sectorial operator and $D\left(A\left(t_{0}, u_{0}\right)\right) \cong D$.

Consider the Cauchy problem for an abstract quasilinear evolution equation

$$
\left\{\begin{array}{l}
u^{\prime}(t)=A(t, u(t)) u(t)+f(t, u(t)), \quad t \in(0, T] \\
u(0)=u_{0}
\end{array}\right.
$$

A classical solution in $[0, T]$ of Problem $(2.1)$ is understood to be a function $u \in C([0, T] ; \mathcal{X}) \cap C((0, T] ; D) \cap C^{1}((0, T] ; \mathcal{X})$ satisfying the equation of $(2.1)$ for every $t \in(0, T]$. Under the hypotheses (i) and (ii), Lunardi [28] established unique existence of local-in-time solution of the problem (2.1).

Proposition 2.1. ([28, Theorem2.1]) For every $\bar{u}_{0} \in U \cap(\mathcal{X}, D)_{\beta, \infty}$ with $\theta<$ $\beta<1$, there exist $R=R\left(\bar{u}_{0}\right)>0$ and $\delta=\delta\left(\bar{u}_{0}\right)>0$ such that, for each $u_{0} \in B\left(\bar{u}_{0}, R\right) \subset(\mathcal{X}, D)_{\beta, \infty}$, there exists a classical solution $u=u(t)$ of Problem $(2.2)$ in $[0, \delta]$, and it is unique in $\bigcup_{\alpha, \sigma>0} B C^{\alpha}\left([0, \delta] ;(\mathcal{X}, D)_{\theta, \infty}\right)$.

By Proposition 2.1 we are able to prove existence and uniqueness of localin-time smooth solution of Problem (1.3)-(1.4) in the "concrete" setting. At this stage we need that initial data should be taken from $B C^{1+\alpha}(\mathbf{R})$ with some $0<\alpha<1$. Setting $u(t)=u(\cdot, t)$, we rewrite the Cauchy problem (1.3)-(1.4) as the abstract Cauchy problem like (2.1) in Banach space $\mathcal{X}=B C(\mathbf{R})$. We set $D=B C^{2}(\mathbf{R})$. It is known then that $(\mathcal{X}, D)_{\theta, \infty} \cong B C^{2 \theta}(\mathbf{R})$ if $1 / 2<\theta<$ 1 ([29]). Here we write $Y \cong Z$ for subspaces $Y$ and $Z$ if $Y \subset Z$ and $Z \subset Y$, 
where the notation " $\subset$ " stands for continuous embedding. Here we choose the constant $\theta \in(1 / 2,1)$ so that $2 \theta=1+\alpha$. We now define a nonlinear function

$$
f(u)=-\frac{n-1}{u}
$$

in an open set $U=\left\{u \in(\mathcal{X}, D)_{\theta, \infty} \mid \inf _{x \in \mathbf{R}} u(x)>0\right\}$ of $(\mathcal{X}, D)_{\theta, \infty}$ and, for every $\varphi \in(\mathcal{X}, D)_{\theta, \infty}$ with $1 / 2<\theta<1$, define a closed operator $A(\varphi): \mathcal{X} \rightarrow \mathcal{X}$ as

$$
A(\varphi) v=\frac{1}{1+\varphi_{x}^{2}} v_{x x}, \quad v \in D(A(\varphi)),
$$

with

$$
D(A(\varphi))=\left\{u \in \bigcap_{p \geq 1} W_{l o c}^{2, p}(\mathbf{R}) \mid u, \frac{1}{1+\varphi_{x}^{2}} u_{x x} \in \mathcal{X}\right\} .
$$

Then $A(\varphi)$ is a sectorial operator in $\mathcal{X}$ and $D(A(\varphi)) \cong D([29$, Corollary 3.1.9]). It is readily seen that $A(\varphi) \in L(D, \mathcal{X})$ and the property (i) and (ii) above hold with $A(t, u) \equiv A(u)$ and $f(t, u) \equiv f(u)$. The problem (1.3)-(1.4) can be rewritten as the abstract Cauchy problem

$$
\left\{\begin{array}{l}
u^{\prime}(t)=A(u(t)) u(t)+f(u(t)), t>0 \\
u(0)=u_{0}
\end{array}\right.
$$

for which we may apply Proposition 2.1. Consequently we obtain

Corollary 2.2. For every $\bar{u}_{0} \in B C^{1+\alpha}(\boldsymbol{R})$ with $0<\alpha<1$ satisfying $\inf _{x \in \boldsymbol{R}} \bar{u}_{0}(x)>$ 0 , there exist $R^{\prime}=R^{\prime}\left(\bar{u}_{0}\right)>0$ and $\delta=\delta\left(\bar{u}_{0}\right)>0$ such that, for each $u_{0} \in B\left(\overline{u_{0}}, R^{\prime}\right) \subset B C^{2 \beta}(\boldsymbol{R})$, there exists a unique $u \in B U C(\boldsymbol{R} \times[0, \delta)) \cap C^{\infty}(\boldsymbol{R} \times$ $(0, \delta))$ satisfying equation (1.3) in $\boldsymbol{R} \times(0, \delta)$ and initial condition $u(x, 0)=u_{0}$ for all $x \in \boldsymbol{R}$.

Proof. For the solution $u(t)$ of (2.2) obtained by Proposition 2.1, we set $u(x, t)=$ $\{u(t)\}(x)$ to observe that it is the desired solution by a standard argument (cf. [28, 29]). Differentiability of $u$ for $t>0$ may be proved by the usual parabolic regularizing argument.

We shall recall some basic results obtained in [1] in the restricted form convenient to our aim. In what follows, the half interval $(0, \infty)$ is denoted by $\mathbf{R}_{+}$. Roughly speaking, the next lemma states that a solution of (1.3) has a bounded gradient provided that the solution itself is bounded away from zero. It allows us to treat initial data which are necessarily smooth and plays a crucial role in the following sections.

Lemma 2.3. Let $u$ be a solution of $(1.3)$ in $(a, b) \times(0, T)$ for some $-\infty<a<$ $b<\infty$. Then there is a function $\sigma: \boldsymbol{R}_{+} \times \boldsymbol{R}_{+} \rightarrow \boldsymbol{R}$ such that

$$
\left|u_{x}(x, t)\right| \leq \sigma(t, u(x, t))
$$

holds for all $a<x<b, 0<t<T$. The function $\sigma$ has the form $\sigma(t, u)=$ $\exp (\rho(u) / t)$ with a positive continuous function $\rho$ on $\boldsymbol{R}_{+}$and depends only on $\sup u(x, 0)$ and $b-a$. Moreover, if $u$ solves the equation in $\boldsymbol{R} \times(0, T)$, then (2.3) holds in $\boldsymbol{R} \times(0, T)$ and $\sigma$ depends only on $\sup u(x, 0)$. 
This lemma is proved in $[1$, Theorem $4.3(\mathrm{~b})]$ with interval $(a, b)$ replaced by a certain time-dependent interval $(a(t), b(t))$ without discussing how $\sigma$ depends on $a$ and $b$. By a careful reading of the proof, however, one may have the same result for an arbitrary fixed interval $(a, b)$ with $\sigma$ depending on $a, b$ only through $b-a$. The last statement is an immediate consequence of the first one, since the whole real line $\mathbf{R}$ is covered by countably many intervals with unit length.

With the aid of Lemma 2.3, we are able to prove local well-posedness of the Cauchy problem (1.3)-(1.4) for any bounded uniformly continuous initial data having positive infimum over the whole $\mathbf{R}$ as in the next proposition.

Proposition 2.4. For every $u_{0}$ satisfying $(H 1)$ and $(H 2)$, there exist $\tau>0$ and a unique $u \in B U C(\boldsymbol{R} \times[0, \tau)) \cap C^{\infty}(\boldsymbol{R} \times(0, \tau))$ satisfying equation (1.3) in $\boldsymbol{R} \times(0, \tau)$ and initial condition (1.4) in $\boldsymbol{R}$.

Proof. First of all, we claim that for any $\nu>0$ and $\delta>0$ small enough, there exists a constant $K>0$ depending only on $n, \nu$ and $\delta$ such that

$$
\|u(t)-v(t)\|_{\infty} \leq K\left\|u_{0}-v_{0}\right\|_{\infty} \quad \text { in }(0, \delta)
$$

for any classical solutions $u$ and $v$ of the Cauchy problem (1.3)-(1.4) in $\mathbf{R} \times(0, \delta)$ with smooth initial data $u_{0}$ and $v_{0}$, respectively and such that

$$
\inf _{(x, t) \in \mathbf{R} \times(0, \delta)}\{u(x, t), v(x, t)\}>\nu .
$$

Uniqueness of classical solutions in $C^{2,1}(\mathbf{R} \times(0, \tau)) \cap B C(\mathbf{R} \times[0, \tau))$ then follows immediately from (2.4). We set $a(\xi)=1 /\left(1+\xi^{2}\right)$ for $\xi \in \mathbf{R}$. By the mean value theorem, we have

$$
\begin{aligned}
a(p) X-a(q) Y=\int_{0}^{1} a^{\prime}(\theta p+(1-\theta) q)( & X+(1-\theta) Y) d \theta(p-q) \\
& +\int_{0}^{1} a(\theta p+(1-\theta) q) d \theta(X-Y) .
\end{aligned}
$$

It is readily seen that $w:=u-v$ satisfies the parabolic equation

$$
w_{t}=A(x, t) w_{x x}+B(x, t) w_{x}+C(x, t) w \quad \text { in } \mathbf{R} \times(0, \delta),
$$

where

$$
\begin{aligned}
A(x, t) & =\int_{0}^{1} a\left(\theta u_{x}+(1-\theta) v_{x}\right) d \theta(>0), \\
B(x, t) & =\int_{0}^{1} a^{\prime}\left(\theta u_{x}+(1-\theta) v_{x}\right)\left(\theta u_{x x}+(1-\theta) v_{x x}\right) d \theta, \\
C(x, t) & =\frac{n-1}{u v} .
\end{aligned}
$$

We may take a constant $\mu>0$ depending only on $n$ and $\nu$ such that $\|C\|_{L^{\infty}(\mathbf{R} \times(0, \delta))} \leq$ $\mu$ by virtue of (2.5). Setting $z=e^{-\mu t} w$, we see that $z_{t} \leq A(x, t) z_{x x}+B(x, t) z_{x}$ from (2.6) and observe that

$$
\sup _{x \in \mathbf{R}} z(x, t) \leq \sup _{x \in \mathbf{R}} z(x, 0)
$$


by the maximum principle for unbounded domain (cf. for instance, [23, chapter 1]). Estimate (2.7) implies then that the claim (2.4) holds with $K=e^{\mu \delta}$.

We then proceed to proving existence of the solution of (1.3)-(1.4) under the hypotheses $(H 1)$ and $(H 2)$. We may take a sequence $\left\{u_{0, j}\right\} \subset B C^{1+\alpha}(\mathbf{R})$ such that

$$
\inf _{x \in \mathbf{R}} u_{0, j}(x)>0 \quad \text { and } \quad u_{0, j} \rightarrow u_{0} \quad \text { in } B C(\mathbf{R}) \text { as } j \rightarrow \infty .
$$

For every $j$, there exist a constant $\delta_{j}>0$ and a unique solution $u_{j} \in B U C(\mathbf{R} \times$ $\left.\left[0, \delta_{j}\right)\right) \cap C^{\infty}\left(\mathbf{R} \times\left(0, \delta_{j}\right)\right)$ of (1.3)-(1.4) with initial data $u_{0, j}$ by Corollary 2.2. Moreover, we have $\inf _{x \in \mathbf{R}} u_{0, j}(x) \geq m / 2$ for $j$ sufficiently large, where $m=$ $\inf _{x \in \mathbf{R}} u_{0}(x)>0$. A comparison argument reveals then that there are positive constants $c_{1}$ and $c_{2}$ such that

$$
c_{1} \leq u_{j}(x, t) \leq c_{2} \quad \text { in } \mathbf{R} \times[0, \tau], \quad j=1,2, \ldots,
$$

whence existence times $\delta_{j}$ are estimated below by a positive constant $\tau$ depending only on $m$ and $n$. We now take advantage of Lemma 2.3 to see that

$$
\left|\left(u_{j}\right)_{x}(x, t)\right| \leq \sigma\left(t, u_{j}(x, t)\right) \leq \exp \left(\rho\left(c_{1}\right) / t\right) \quad \text { in } \mathbf{R} \times(0, \tau) .
$$

It follows from (2.8) and gradient Hölder estimate (cf. [27, Chapter VI, Theorem 1.1]) that for any $a>0, \tau^{\prime} \in(0, \tau)$ and $\alpha \in(0,1)$, there exists a positive constant $G$ depending only on $c_{1}, c_{2}, n, \tau, \tau^{\prime}, a$ and $\alpha$ such that

$$
\sup _{j \geq 1}\left\|u_{j}\right\|_{B C^{1+\alpha,(1+\alpha) / 2}\left((-a, a) \times\left(\tau^{\prime}, \tau\right]\right)} \leq G .
$$

Schauder's interior estimate $([10,27])$ then implies that the sequence $\left\{u_{j}\right\}$ is bounded in $B C^{2+\alpha, 1+\alpha / 2}(Q)$ for each $Q \Subset(-a / 2, a / 2) \times\left(\tau^{\prime}, \tau\right]$. Hence $u_{j}$ is convergent to some positive function $u$ defined on the whole $\mathbf{R}$ as $j \rightarrow \infty$ in $C_{l o c}^{2+\beta, 1+\beta / 2}\left(\mathbf{R} \times\left(\tau^{\prime}, \tau\right]\right)$ with any $\beta \in(0, \alpha)$. Moreover, we may prove that $u$ is infinitely differentiable in $\mathbf{R} \times(0, \tau]$, using Schauder's interior estimate (cf. the proof of Theorem 1.3 at the end of the next section). Since $\tau^{\prime}$ is arbitrary and $u_{j} \in B U C(\mathbf{R} \times[0, \tau])$, we see that $u$ belongs to $C^{\infty}(\mathbf{R} \times(0, \tau]) \cap B U C(\mathbf{R} \times$ $[0, \tau])$ and satisfies equation (1.3) and initial condition (1.4). The proof is now complete.

We close this section by recalling a useful tool from [1] to investigate shape of axisymmetric hypersurfaces $\Gamma(t)$ evolved by the mean curvature flow (1.1). To state it, we need to introduce some notations. As is proved in [1, Theorem $4.3(\mathrm{a})$ ], if initial hypersurface $\Gamma(0)$ is given by rotating the graph of a function around the $x$-axis, then so are $\Gamma(t), t>0$, as long as they exist. The number of necks of $\Gamma(t)$ over a closed interval $I=[a, b]$ is a finite nonincreasing function of time provided that there is no neck on $\partial I$. It follows that, after a while, the number of necks remain constant and accordingly we may even assume that hypersurface $\Gamma(t)$ have $m \geq 0$ necks. Then $x \mapsto u(x, t)$ will have $m$ local minima and $m+1$ maxima in every bounded interval and the number is nonincreasing in time (unless they are on the boundary). Their location are denoted by $\left\{\xi_{j}(t)\right\}_{1 \leq j \leq m}$ and $\left\{\eta_{j}(t)\right\}_{0 \leq j \leq m}$, respectively, ordered them so that

$$
\eta_{0}(t)<\xi_{1}(t)<\eta_{1}(t)<\cdots<\xi_{m}(t)<\eta_{m}(t)
$$


Then [1, Converging Necks Lemma 5.1] guarantees that even when the solution becomes singular at some time $T$, the $\operatorname{limits}_{\lim _{t} T} \xi_{j}(t)=\xi(T)$ and $\lim _{t \nearrow T} \eta_{j}(t)=\eta(T)$ exist for each $j$. Moreover, it follows from [1, Single-Point Pinching Lemma 5.2], which we recall just below for readers' convenience, that there is no quenching point between $\xi_{j}(T)$ and $\eta_{j}(T)$ for every $j$.

Lemma 2.5. If $\eta_{j-1}(T)<\xi_{j-1}(T)$, then $u(x, t)$ is bounded from below uniformly in $t \in(0, T)$, for all $x \in\left(\eta_{j-1}(T), \xi_{j-1}(T)\right)$; i.e., for any compact interval $[c, d] \subset\left(\eta_{j-1}(T), \xi_{j-1}(T)\right)$ there exists a $\delta>0$ such that $u(x, t) \geq \delta$ for any $x \in[c, d], t \in(0, T)$.

\section{Profile at minimal quenching time}

In this section we prove Theorem 1.3. We just recall here some notation previously introduced in $\S 1$. Initial data $u_{0} \in B U C(\mathbf{R})$ is such that $m:=$ $\inf _{x \in \mathbf{R}} u_{0}(x)>0$. The function $v_{m}=v_{m}(t)$ denotes a solution of (1.3) with initial data $m$. It is nothing but a solution of ODE obtained by getting rid of the term $u_{x x} /\left(1+u_{x}^{2}\right)$ from (1.3). Its quenching time is denoted by $T(m)$. It gives a lower bound for the quenching times $T\left(u_{0}\right)$ of all solutions of (1.3). We begin with investigating a necessary condition for a solution of (1.3)-(1.4) to have a minimal quenching time, that is, a necessary condition in order that $T\left(u_{0}\right)=T(m)$.

Proposition 3.1. Suppose that a solution $u$ of the Cauchy problem (1.3)-(1.4) has a minimal quenching time $T(m)$. Then

$$
\liminf _{x \rightarrow-\infty} u(x, t)=v_{m}(t) \quad \text { or } \quad \liminf _{x \rightarrow+\infty} u(x, t)=v_{m}(t)
$$

for every $t \in[0, T(m))$.

Proof. We argue by contradiction. Since the claim is obvious when $u_{0} \equiv m$, we may hereafter assume that $u_{0} \not \equiv m$. Suppose contrary to the conclusion that there were $t_{0} \in[0, T(m))$ such that

$$
\ell_{-\infty}:=\liminf _{x \rightarrow-\infty} u\left(x, t_{0}\right)>v_{m}\left(t_{0}\right) \quad \text { and } \quad \ell_{+\infty}:=\liminf _{x \rightarrow+\infty} u\left(x, t_{0}\right)>v_{m}\left(t_{0}\right) .
$$

We set $\ell_{0}:=\min \left\{\ell_{-\infty}, \ell_{+\infty}\right\}$. Then for any $v_{m}\left(t_{0}\right)<\tilde{\ell}_{0}<\ell_{0}$, there is an $R>0$ such that

$$
u\left(x, t_{0}\right) \geq \tilde{\ell}_{0} \quad \text { for all }|x| \geq R .
$$

We shall begin with the case of $t_{0}>0$. By the strong maximum principle, we have

$$
u\left(x, t_{0}\right)>v_{m}\left(t_{0}\right) \quad \text { in } \mathbf{R},
$$

since otherwise $u_{0} \equiv m$. It follows from (3.1) and (3.2) that

$$
u\left(x, t_{0}\right) \geq \ell>v_{m}\left(t_{0}\right) \quad \text { in } \mathbf{R},
$$

where $\ell:=\inf _{-\infty<x<+\infty} u\left(x, t_{0}\right)$. A comparison argument yields then that

$$
u(x, t) \geq v_{\ell}\left(t-t_{0}\right)>v_{m}(t) \quad \text { in } \mathbf{R} \times\left(t_{0}, t_{0}+T(\ell)\right) .
$$


Thus $T\left(u_{0}\right) \geq t_{0}+T(\ell)>t_{0}+T\left(v_{m}\left(t_{0}\right)\right)=T(m)$, which contradicts the assumption that the quenching time of $u$ is minimal, that is, $T\left(u_{0}\right)=T(m)$.

We shall proceed to the case of $t_{0}=0$. Take a uniformly continuous function $\underline{u_{0}}$ having the following properties:

$\underline{u_{0}}$ is even and nonincreasing (resp. nondecreasing) in $x<0$ (resp. $x>0$ );

$\underline{u_{0}} \leq u_{0} \quad$ in $\mathbf{R}$;

$\underline{u_{0}} \equiv m$ in $[-R-1, R+1]$ and $\liminf _{x \rightarrow \pm \infty} \underline{u_{0}}(x)=\ell_{0}$.

Denote by $\underline{u}$ the solution of equation (1.3) with initial data replaced by $u_{0}$. Then, by the comparison principle, we have

$$
v_{m}(t) \leq \underline{u} \leq u \quad \text { in }(-\infty,+\infty) \times(0, T(m))
$$

Combining the assumption that $T\left(u_{0}\right)=T(m)$ with $(3.3)$, we see that $T\left(\underline{u_{0}}\right)=$ $T(m)$.

We then claim that the solution $\underline{u}(\cdot, t)$ is an even function and nonincreasing (resp. nondecreasing) for $x<0$ (resp. $x>0$ ) for each $t \in(0, T(m))$. To show this, we appeal to a standard reflection argument. For any $\lambda>0$ we set $\bar{u}(x, t):=\underline{u}(2 \lambda-x, t)$ and observe that it is a solution of (1.3)-(1.4) with initial data $\bar{u}(x, 0)=\underline{u}_{0}(2 \lambda-x)$. Since $\underline{u}_{0}(x) \leq \underline{u}_{0}(2 \lambda-x)$ for any $x<\lambda$ by assumption and $\bar{u}(\lambda, t)=\underline{u}(\lambda, t)$ for any $t \in(0, T(m))$, we may apply the comparison principle to deduce that

$$
\underline{u}(x, t) \leq \bar{u}(x, t) \text { in }(-\infty, \lambda) \times(0, T(m)),
$$

which simultaneously implies that $\underline{u}(\cdot, t)$ is nondecreasing for $x>0$. A similar argument shows that $\underline{u}(\cdot, t)$ is nonincreasing for $x<0$. Uniqueness of solution forces $\underline{u}$ to be even in $x$.

Since $\underline{u}(x, t) \geq \underline{u}(0, t)>v_{m}(t)$ for any $0<t<T(m)$ by the properties on $\underline{u}$ mentioned above and the strong maximum principle, we observe that

$$
\underline{\ell}:=\inf _{x \in \mathbf{R}} u\left(x, t_{1}\right) \geq \underline{u}\left(0, t_{1}\right)>v_{m}\left(t_{1}\right) \quad \text { for any } t_{1} \in(0, T(m)) .
$$

Having obtained (3.4), we reach at a contradiction in the same way as in the first case. The proof is now complete.

From Proposition 3.1, we see that the solution $u$ of (1.3)-(1.4) with a minimal quenching time necessarily quenches at space infinity in the sense of (1.11) with $T\left(u_{0}\right)=T(m)$.

Corollary 3.2. Suppose that a solution $u$ of the Cauchy problem (1.3)-(1.4) quenches at minimal quenching time $T(m)$. Then it quenches at $x=-\infty$ or $+\infty$.

We see that $u\left(x, t_{0}\right)>v_{m}\left(t_{0}\right)$ in $\mathbf{R}$ for any $t_{0} \in(0, T(m))$ unless $u_{0}$ is a constant by the strong maximum principle. Let $a>0$ be any constant. We construct a subsolution of (1.3)-(1.4) in $(-a, a) \times\left(t_{0}, T(m)\right)$. Let $w_{0} \in C^{2}(-a, a)$ be a positive even function satisfying

$$
\begin{aligned}
& w_{0}^{\prime \prime}(x)<0, w_{0}^{\prime}(0)=0, \text { for } x \in(-a, a), \\
& v_{m}\left(t_{0}\right) \leq w_{0} \leq u\left(x, t_{0}\right) \text { in }(-a, a), w_{0}( \pm a)=v_{m}\left(t_{0}\right) .
\end{aligned}
$$


Let us consider the following initial-boundary value problem:

$$
\left\{\begin{array}{l}
w_{t}=\frac{w_{x x}}{1+\left(w_{x}\right)^{2}}-\frac{n-1}{w}, \quad \text { in }(-a, a) \times\left(t_{0}, T(m)\right), \\
w( \pm a, t)=v_{m}(t), \text { for } t \in\left(t_{0}, T(m)\right), \\
w\left(x, t_{0}\right)=w_{0}(x), \text { in }(-a, a) .
\end{array}\right.
$$

Lemma 3.3. Assume that $w_{0}$ satisfies (3.5). Then the solution $w(x, t)$ of $(3.6)$ is an even function with respect to $x$ in $(-a, a)$ and attains its maximum at $x=0$ for each $t \in(0, T(m))$. Moreover,

$$
w_{x}>0 \text { in }(-a, 0) \times(0, T(m)) \text { and } w_{x}<0 \text { in }(0, a) \times(0, T(m)) .
$$

Proof. We appeal to a standard reflection argument as was used in the proof of Proposition 3.1, but we should discuss more precisely to show the strict monotonicity (3.7). Let $\lambda \in[0, a)$ and denote by $w^{\lambda}$ the reflection of $w$ in the point $x=\lambda$, that is, $w^{\lambda}(x, t)=w(2 \lambda-x, t)$. Since the equation is reflection invariant, if $w$ solves the equation of $(3.6)$ in $(\lambda, a) \times\left(t_{0}, T(m)\right)$ [or in $(-a,-\lambda) \times$ $\left.\left(t_{0}, T(m)\right)\right], w^{\lambda}$ solves the same equation in $(-a+2 \lambda, \lambda) \times\left(t_{0}, T(m)\right)$ [or in $\left.(-\lambda, a-2 \lambda) \times\left(t_{0}, T(m)\right)\right]$. In particular, if $w(x, t)$ is a solution of $(3.6)$, so is $w(-x, t)$. By assumption, $w_{0}(x)=w_{0}(-x)$ in $(-a, a)$. Uniqueness of solution implies then that $w(-x, t)=w(x, t)$ in $(-a, a) \times\left(t_{0}, T(m)\right)$ and the first assertion is concluded.

We next prove that $w_{x}>0$ in $(-a, 0) \times\left(t_{0}, T(m)\right)$. For $\lambda \in(0, a)$, we set $\Psi:=w^{\lambda}-w$ and observe that it satisfies a parabolic equation of the form

$$
\Psi_{t}=A(x, t) \Psi_{x x}+B(x, t) \Psi_{x}+C(x, t) \Psi \quad \text { in }(\lambda, a) \times\left(t_{0}, T(m)\right),
$$

where the coefficients $A, B, C$ are continuous functions and that $\Psi(\lambda, t)=0$ for $t \in\left(t_{0}, T(m)\right)$. By $(3.5), \Psi\left(x, t_{0}\right) \geq 0$ and $\not \equiv 0$ in $(\lambda, a)$. The strong maximum principle then forces $\Psi>0$ in $(\lambda, a) \times\left(t_{0}, T(m)\right)$. We thus obtain, by Hopf's boundary lemma, that

$$
-\Psi_{x}(\lambda, t)<0 \text { for } t_{0}<t<T(m)
$$

or equivalently, $w_{x}(\lambda, t)<0$ for $t_{0}<t<T(m)$. Similarly we obtain $w_{x}(-\lambda, t)>$ 0 for $t_{0}<t<T(m)$. Since $\lambda$ is arbitrary, the assertion (3.7) is concluded and consequently $w(\cdot, t)$ takes its maximum at $x=0$ for each $t \in\left(t_{0}, T(m)\right)$. The proof is now complete.

Lemma 3.4. Assume the same hypothses with Lemma 3.3. Then the solution $w$ of problem (3.6) has no quenching point in $(-a, a)$ at $t=T(m)$.

Proof. By Lemma 3.3, $w_{x}(x, t) \neq 0$ except $x=0$ for each $t \in\left(t_{0}, T(m)\right)$. We may apply Lemma 2.5, to get a positivity of the infimum of $w$ in $(b, c) \times\left(t_{0}, T(m)\right)$ for any $-a<b<c<a$. Indeed, if there were a quenching point in $(-a, a), w$ is forced to quench identically by (3.7), which contradicts Lemma 2.5. Hence $w$ has no quenching point in $(-a, a)$, which completes the proof.

Proof of Theorem 1.3. Let $a, t_{0}, w$ be as in Lemma 3.4. By the comparison theorem, we have $u(x, t) \geq w(x, t)$ in $(-a, a) \times\left(t_{0}, T(m)\right)$. Lemma 3.4 implies that $w$ has a positive infimum in every compact subinterval of $(-a, a)$ up to 
$t=T(m)$, which simultaneously guarantees that $u$ has no quenching point in $(-a, a)$. Since $a$ is arbitrary, we see that there is no quenching point in the whole real line $\mathbf{R}$.

We shall show the latter statement. Let $K$ be a bounded closed interval. From what we have proved above, the infimum of $u$ in $Q:=K \times(0, T(m))$ is strictly positive, and thus $u_{x}$ is bounded there by Lemma 2.3. It then follows from [27, Chapter VI, Theorem 1.1] that Hölder norm of $u_{x}$ with any exponent $\alpha \in(0,1)$ is bounded by a constant in $Q^{\prime}:=K^{\prime} \times(T(m) / 4, T(m))$, where $K^{\prime}$ is any interval such that $K^{\prime} \Subset K$. The constant depends only on $m, n, \alpha$, the bound of $u_{x}$ and the distance between $Q$ and $Q^{\prime}$. We shall now regard the equation (1.3) as the linear equation

$$
u_{t}=a(x, t) u_{x x}+b(x, t) u, \quad x \in K, t>0
$$

with bounded coefficients $a(x, t)=1 /\left(1+u_{x}^{2}(x, t)\right)$ and $b(x, t)=-(n-1) / u^{2}(x, t)$. Schauder's interior estimate then implies that $u$ is bounded in $B C^{2+\alpha, 1+\alpha / 2}\left(K^{\prime \prime} \times\right.$ $(T(m) / 2, T(m))$ for any $K^{\prime \prime} \Subset K^{\prime}$. In particular, $u_{t}$ is bounded there and hence the limit $u(x, T(m))=\lim _{t \nearrow T(m)} u(x, t)$ exists. Moreover, for any sequence $\left\{t_{k}\right\} \nearrow T(m),\left\{u\left(\cdot, t_{k}\right)\right\}$ forms a Cauchy sequence in $C^{2+\alpha}(\bar{D})$ for any open interval $D \Subset K$, so that $u(\cdot, T(m)) \in C^{2+\beta}(K)$ for every $0<\beta<\alpha$. Since $K$ was chosen arbitrary, it follows that $u(\cdot, T(m)) \in C^{2+\alpha}(\mathbf{R})$ and $u(\cdot, t)$ is convergent to $u(\cdot, T(m))$ in $C_{\text {loc }}^{2+\alpha}(\mathbf{R})$ for any $\alpha \in(0,1)$. Differentiating the equation for $u$ by $x$ and invoking Schauder's interior estimate many times, we obtain further regularity on $u(\cdot, T(m))$ to see $u(\cdot, T(m)) \in C^{\infty}(\mathbf{R})$ eventually and $u(\cdot, t) \rightarrow u(\cdot, T(m))$ as $t \nearrow T(m)$ in $C^{\infty}(\mathbf{R})$ (endowed with the usual seminorms). The other assertions on $u(x, T(m))$ are easily obtained from the former statement and Proposition 3.1. The proof is now complete.

\section{A necessary and sufficient condition for minimal quenching time}

In this section we prove Theorem 1.4. Proposition 3.1 shows a necessary condition for a solution of the Cauchy problem (1.3)-(1.4) to have a minimal quenching time. However, it is not a sufficient condition. We establish a necessary and sufficient condition in the following two subsections.

\subsection{A sufficient condition for minimal quenching time}

In this subsection we will prove that condition (1.7) forces a solution to quench at minimal quenching time. Theorem 1.1 is concluded immediately as a consequence of the following proposition.

Proposition 4.1. Assume that an initial datum $u_{0}$ satisfies (1.9) (or (1.10)). Then the solution $u$ of (1.3)-(1.4) has a minimal quenching time. Moreover, for any $\varepsilon, T \in(0, T(m))$ and $R>0$,

$$
\lim _{k \rightarrow \infty} \sup _{|x| \leq R, \varepsilon \leq t \leq T}\left|u\left(x+x_{k}, t\right)-v_{m}(t)\right|=0,
$$

where the sequence $\left\{x_{k}\right\}$ is as in condition (1.9) (or (1.10)). 
Proof. We prove the claim only when $u_{0}$ fulfills condition (1.9), since the other case is similarly proved. For a sequence $\left\{x_{k}\right\}$ satisfying (1.9), we set

$$
W_{k}(x, t)=u\left(x+x_{k}, t\right)-v_{m}(t), \quad k=1,2, \ldots
$$

and then observe that each of them solves the linear parabolic equation

$$
W_{t}=a_{k}(x, t) W_{x x}+b_{k}(x, t) W
$$

where $a_{k}(x, t)=1 /\left(1+u_{x}\left(x+x_{k}, t\right)^{2}\right)$ and $b_{k}(x, t)=1 /\left(u\left(x+x_{k}, t\right) v_{m}(t)\right)$. Let $G_{k}$ denote fundamental solutions of the Cauchy problems for $(4.2), k=1,2, \ldots$ Then each $W_{k}$ satisfies

$$
W_{k}(x, t)=\int_{-\infty}^{\infty} G_{k}(x, t ; y, 0)\left\{u_{0}\left(y+x_{k}\right)-m\right\} d y
$$

Notice that for any $\varepsilon, T \in(0, T(m))$, there is a constant $\lambda_{1}>0$ such that

$$
\lambda_{1} \leq a_{k}(x, t) \leq 1 \quad \text { in } \mathbf{R} \times[\varepsilon, T],
$$

since we have the estimate for $\left\|u_{x}(\cdot, t)\right\|_{\infty}$ for $t \leq T$ due to Lemma 2.3. Hence each $G_{k}$ is estimated from above as

$$
\begin{aligned}
& G_{k}(x, t ; y, 0) \leq \frac{c_{1}}{t^{1 / 2}} \exp \left(-\frac{c_{2}|x-y|^{2}}{t}\right) \\
&-\infty<x, y<\infty, \quad \varepsilon \leq t \leq T,
\end{aligned}
$$

where $c_{1}$ and $c_{2}$ are positive constants which depend on $m, T$ and $\lambda_{1}$, but are independent of $k$. (See e.g., [10, Chapter 1].) From (4.3), (4.4) and the comparison theorem, we have

$$
0 \leq W_{k}(x, t) \leq \frac{c_{1}}{t^{1 / 2}} \int_{-\infty}^{\infty} \exp \left(-\frac{c_{2}|x-y|^{2}}{t}\right)\left\{u_{0}\left(y+x_{k}\right)-m\right\} d y
$$

for $-\infty<x<\infty$ and $\varepsilon \leq t \leq T$. By the definition of the sequence $\left\{x_{k}\right\}$ and the Lebesgue convergence theorem, we then see that $\lim _{k \rightarrow \infty} W_{k}(x, t)=0$ and the convergence is uniform in the region of the form $\{(x, t)|| x \mid \leq R, \varepsilon \leq t \leq T\}$ with any $R>0$, or equivalently,

$$
\lim _{k \rightarrow \infty} \sup _{|x| \leq R, \varepsilon \leq t \leq T}\left|u\left(x+x_{k}, t\right)-v_{m}(t)\right|=0 .
$$

The proof is now complete.

Proof of Proposition 1.2. Proposition 1.2 is an immediate consequence of Proposition 4.1.

In view of Proposition 4.1, we see that conditions (1.17) or (1.18), which are much weaker than (1.7) or (1.8), are sufficient conditions for a solution of the Cauchy problem (1.3)-(1.4) with initial data $u_{0}$ to have a minimal quenching time $T(m)$. In fact, these conditions are also necessary, as we will prove in the next subsection. 


\subsection{A necessary condition for minimal quenching time}

The goal in this subsection is to prove that the conditions (1.17) or (1.18) stated with the weighted mean value function $A_{\rho}\left(x ; u_{0}\right)$ in $(1.16)$ are necessary conditions on initial data for a solution of (1.3)-(1.4) to have a minimal quenching time. Namely,

Proposition 4.2. Let $u$ be a solution of the Cauchy problem (1.3)-(1.4) with initial data $u_{0}$ having a minimal quenching time $T(m)$. Then the initial data $u_{0}$ satisfies condition (1.17) or (1.18).

We need a few lemmata to prove Proposition 4.2.

Lemma 4.3. Assume the same hypotheses with Proposition 4.2. Suppose that

$$
A_{\rho}\left(0 ; u_{0}\right) \geq \ell
$$

for some $\ell>m$. Then there exists a positive constant $R_{0}$ depending only on $\sup _{x \in \boldsymbol{R}} u_{0}(x), \ell$ and $m$ such that

$$
\left(\int_{-R_{0}}^{R_{0}} \rho(y) d y\right)^{-1} \int_{-R_{0}}^{R_{0}} \rho(-y) u_{0}(y) d y \geq \frac{\ell+m}{2}=: \ell_{1}(>m) .
$$

Moreover, there exist $x_{0} \in\left[-R_{0}, R_{0}\right]$ and $r_{0} \in\left(0, R_{0}\right)$ depending only on $\ell_{1}, \sup u_{0}, m$ and $R_{0}$ such that

$$
u_{0}(x) \geq \frac{\ell_{1}+m}{2} \quad \text { for }\left|x-x_{0}\right|<r_{0} .
$$

Proof. Since

$$
1=\int_{-\infty}^{\infty} \rho(y) d y=\left(\int_{-\infty}^{-R}+\int_{-R}^{R}+\int_{R}^{\infty}\right) \rho(y) d y=: I_{1}+I_{2}+I_{3},
$$

we have

$$
\frac{1}{I_{2}} \int_{-R}^{R} \rho(-y) u_{0}(y) d y \geq-\left(I_{1}+I_{3}\right) \sup _{y \in \mathbf{R}} u_{0}(y)+A_{\rho}\left(0 ; u_{0}\right) .
$$

Taking $R=R_{0}$ sufficiently large, we observe that

$$
\left(I_{1}+I_{3}\right) \sup _{y \in \mathbf{R}} u_{0}(y) \leq \frac{\ell-m}{2}
$$

since $\rho \in L^{1}(\mathbf{R})$. It follows from (4.9) and (4.10) that

$$
\left(\int_{-R_{0}}^{R_{0}} \rho(y) d y\right)^{-1} \int_{-R_{0}}^{R_{0}} \rho(-y) u_{0}(y) d y \geq-\frac{\ell-m}{2}+\ell=\ell_{1} .
$$

We thus conclude (4.7). Having obtained (4.7), we readily see that there exists $x_{0} \in\left[-R_{0}, R_{0}\right]$ such that

$$
u_{0}\left(x_{0}\right) \geq \ell_{1} .
$$

On the other hand, there is a positive constant $\delta=\delta(\ell, m)$ such that

$$
\left|u_{0}\left(x_{1}\right)-u_{0}\left(x_{2}\right)\right| \leq \frac{\ell_{1}-m}{2} \quad \text { provided }\left|x_{1}-x_{2}\right|<\delta
$$

by the assumption of uniform continuity of $u_{0}$ in $(H 1)$. We thus obtain (4.8) with $r_{0}=\min \left\{\delta, R_{0} / 2\right\}$ from (4.11) and (4.12), which completes the proof. 
Let $\ell_{1}, r_{0}$ and $R_{0}$ be as in Lemma 4.3 and let $w_{0}$ be an even bell-shaped continuous function in $\left[-R_{0}-1, R_{0}+1\right]$ satisfying

$$
w_{0}(x)\left\{\begin{array}{l}
=m, \quad \text { if } r_{0} \leq|x| \leq R_{0}+1, \\
\leq \ell_{1}, \quad \text { if } r_{0} / 2 \leq|x|<r_{0} \\
=\ell_{1}, \quad \text { if }|x| \leq r_{0} / 2
\end{array}\right.
$$

Consider the following problem:

$$
\left\{\begin{array}{l}
w_{t}=\frac{w_{x x}}{1+w_{x}^{2}}-\frac{n-1}{w}, \text { in }\left(-R_{0}-1, R_{0}+1\right) \times(0, T(m)) \\
w\left( \pm\left(R_{0}+1\right), t\right)=v_{m}(t), \text { for }(0, T(m)) \\
w(x, 0)=w_{0}(x), \text { in }\left(-R_{0}-1, R_{0}+1\right) .
\end{array}\right.
$$

Lemma 4.4. Let $w$ be a solution of (4.14). Then

$$
\inf _{-R_{0} \leq x \leq R_{0}, 0<t<T(m)} w(x, t)>0 .
$$

Proof. Since it may be proved in the same way as Lemmata 3.3 and 3.4, we omit the precise.

Proof of Proposition 4.2. We prove its contraposition. Namely, we prove that if an initial datum $u_{0}$ does not satisfy (1.17) nor (1.18), then the quenching time of the corresponding solution $u$ is no longer minimal, i.e, $T\left(u_{0}\right)>T(m)$.

Suppose that $u_{0}$ does not satisfy (1.17) nor (1.18). Then there exists $\ell>m$ such that

$$
\inf _{x \in \mathbf{R}} A_{\rho}\left(x ; u_{0}\right) \geq \ell .
$$

For any $a \in \mathbf{R}$ we set $u_{a}(x, t)=u(x+a, t)$ and observe that it is the solution of (1.3) with initial data $u_{0 a}(x):=u_{0}(x+a)$. Moreover, we have

$$
A_{\rho}\left(0 ; u_{0 a}\right)=A_{\rho}\left(a ; u_{0}\right) \geq \ell
$$

from (4.15). Applying Lemma 4.3, we obtain (4.8) with $u_{0}$ replaced by $u_{0 a}$. A comparison argument reveals that

$$
u_{a}(x, t) \geq w(x, t) \quad \text { in }\left(-R_{0}-1, R_{0}+1\right) \times(0, T(m)),
$$

where $w$ is the solution of (4.14). Application of Lemma 4.4 to $u_{a}$ then implies that

$$
u(a, t) \geq \inf _{-R_{0} \leq x \leq R_{0}} u_{a}(x, t) \geq \inf _{-R_{0} \leq x \leq R_{0}, 0<t<T(m)} w(x, t)=: C_{\ell, m}>0,
$$

whence

$$
\inf _{x \in \mathbf{R}} u(x, t) \geq C_{\ell, m} \quad \text { for } 0<t<T(m),
$$

which simultaneously implies that the infimum of $u$ in the whole $\mathbf{R}$ at $t=T(m)$ is strictly positive; $T\left(u_{0}\right)>T(m)$. The proof is now complete.

Proof of Theorem 1.4. Theorem 1.4 immediately follows from Propositions 4.1 and 4.2 . 


\section{$5 \quad$ Profile after quenching}

In this section we assume the conditions (1.7) and (1.8). To study behavior of an evolving hypersurfaces $\Gamma_{t}$ after the quenching (pinching) time we recall a level set solution. We say a closed set $E$ in $\mathbf{R}^{n+1} \times[0, \infty)$ is a level set solution of (1.1) with closed initial data $E_{0} \subset \mathbf{R}^{n+1}$ if there is a viscosity solution $\psi \in B U C\left(\mathbf{R}^{n+1} \times[0, T)\right)$ for all $T>0$ of

$$
\psi_{t}-|\nabla \psi| \nabla \cdot\left(\frac{\nabla \psi}{|\nabla \psi|}\right)=0 \quad \text { in } \mathbf{R}^{n+1} \times(0, T)
$$

with

$$
E=\left\{(X, t) \in \mathbf{R}^{n+1} \times[0, \infty) \mid \psi(X, t) \geq 0\right\}, \quad E(0)=E_{0},
$$

where $E(t)$ is the cross-section of $E$ at time $t$, i.e.,

$$
E(t)=\left\{X \in \mathbf{R}^{n+1} \mid(X, t) \in E\right\} .
$$

For our problem we take

$$
E_{0}=\left\{\left(x, y_{1}, . ., y_{n}\right) \mid r \leq u_{0}(x)\right\}
$$

It is well-known [13] that $\partial E(t)$ is smooth and agrees with the classical solution of $(1.1)$, i.e.,

$$
\partial E(t)=\Gamma_{t}=\left\{\left(x, y_{1}, . ., y_{n}\right) \mid r=u(x, t)\right\}
$$

before the quenching time, i.e., $t<T(m)$. By the left continuity of $E(t)$ in time [13] our Theorem 1.3 implies that $\Gamma_{T(m)}=\partial E(T(m))$ is also smooth. We are able to prove that the open ends of $\Gamma_{T(m)}$ will be closed for $t>T(m)$.

Proposition 5.1. The set $E(t)$ becomes bounded for $t>T(m)$.

Proof. Clearly, $E(t)$ is in a cylinder of radius $\sup u(x, T(m))$. For sufficiently large $x$, the motion of $E(t)$ for $(T(m)-\delta, T(m))$ is monotone. Since $u(x, T(m))$ tends to zero as $x$ tends to infinity, by comparison with the self-similar shrinking doughnut as in [1] we observe that for any $t_{1}>T(m)$ there exists $R_{1}$ such that, the set $E\left(t_{1}\right) \cap\left\{\left(x, y_{1}, . ., y_{n}\right)|| x \mid>R_{1}\right\}$ does not contain the $x$-axis. Thus $E(t)$ is bounded for $t>T(m)$.

We do not discuss whether $E$ is regular, i.e., $E=\bar{D}$ after the quenching time, where $D=\{\psi>0\}$. In other words, we do not discuss whether or not evolution has a fattening phenomenon $[8,13]$.

Acknowledgement. The authors are sincerely grateful to Dr. Takeshi Ohtsuka for reading manuscripts and giving valuable advice.

The work of Y. Giga was partly supported by the Grant-in-Aid for Scientific Reserch, No. 20654017, No.18204011, the Japan Society of the Promotion of Science (JSPS) and by COE Program "Mathematics of Nonlinear Structures via Singularities" sponsored by JSPS. Research by Y. Seki was supported by Research Fellowships of JSPS. Much of the work of N. Umeda was done while he visited the University of Tokyo during 2005-2009 as a postdoctoral fellow. Its hospitality is gratefully acknowledged as well as support from formation of COE "New Mathematical Development Center to Support Scientific Technology" during 2005-2008 and COE "The research and training center for new development in mathematics" in 2009, supported by JSPS. 


\section{References}

[1] S. Altschuler, S. Angenent and Y. Giga, (1995). Mean curvature flow through singularities for surfaces of rotation, J. Geom. Anal. 5, 293-358.

[2] S. B. Angenent and J. J. L. Velázquez, (1997). Degenerate neckpinches in mean curvature flow, J. Reine Angew. Math. 482, 15-66.

[3] K. A. Brakke, 1978. The motion of a surface by its mean curvature, Mathematical Notes, 20. Princeton University Press, Princeton, N.J.

[4] Y. G. Chen, Y. Giga and S. Goto, (1991). Uniqueness and existence of viscosity solutions of generalized mean curvature flow equations, J. Differential Geom. 33, 749-786.

[5] M. G. Crandall, H. Ishii, and P. L. Lions, (1992). User's guide to viscosity solutions of second order partial differential equations, Bull. Amer. Math. Soc. (N.S.) 27, 1-67.

[6] G. Dziuk and B. Kawohl, (1991). On rotationally symmetric mean curvature flow, J. Differential Equations 93, 142-149.

[7] K. Ecker, 2004. Regularity theory for mean curvature flow, Progress in Nonlinear Differential Equations and their Applications, 57. Birkhäuser Boston, Inc., Boston, MA.

[8] L. C. Evans and J. Spruck, (1991). Motion of level sets by mean curvature. I, J. Differential Geom. 33, 635-681.

[9] M. Fila, B. Kawohl and H. A. Levine, (1992). Quenching for quasilinear equations, Comm. Partial Differential Equations 17, 593-614.

[10] A. Friedman, 1964. Partial Differential Equations of Parabolic Type, Prentice-Hall, Englewood Cliffs, N.J.

[11] M. E. Gage and R. S. Hamilton, (1986). The heat equation shrinking convex plane curves, J. Differential Geom. 23, 69-96.

[12] Y. Giga, (1995). The method of level surfaces in connection with the equation of evolution of a surface, (Japanese) Sugaku 47, 321-340; translation in Sugaku Expositions 10(1997), 217-241.

[13] Y. Giga, 2006. Surface Evolution Equations. A level set approach, Birkhäuser, Basel.

[14] M.-H. Giga, Y. Giga and J. Saal, Nonliear Partial Differential Equations - Asymptotic Behaviour of Solutions and Self-Similar solutions, Progress in Math. Birkhäuser, to appear (expanded from original Japanese version M.H. Giga and Y. Giga, Nonliear Partial Differential Equations - Asymptotic Behaviour of Solutions and Self-Similar Solutions, Kyoritsu, Tokyo 1999.)

[15] Y. Giga, M. Ohnuma and Sato, (1999). On the strong maximum principle and the large time behavior of generalized mean curvature flow with the Neumann boundary condition, J. Differential Equations 154, 107-131. 
[16] Y. Giga, Y. Seki and N. Umeda, (2008). Blow-up at space infinity for nonlinear heat equations, Recent Advances in Nonlinear Analysis, World Scientific Publishing, 77-94. (also in EPrint Series of Department of Mathematics, Hokkaido University, 2007.)

[17] Y. Giga and N. Umeda, (2006). On blow-up at space infinity for semilinear heat equations, J. Math. Anal. Appl. 316, 538-555.

[18] Y. Giga and N. Umeda, (2005). Blow-up directions at space infinity for solutions of semilinear heat equations, Bol. Soc. Paran. Mat. 23, 9-28: Correction is avairable in Bol. Soc. Paran. Mat. 24(2006), 19-24.

[19] A. Gladkov, (1992). The behavior as $x \rightarrow \infty$ of solutions of semilinear parabolic equations, (Russian) Mat. Zametki 51, 29-34, 156; translation in Math. Notes 51, 124-128.

[20] M. A. Grayson, (1987) The heat equation shrinks embedded plane curves to round points, J. Differential Geom. 26, 285-314.

[21] M. A. Grayson, (1989). A short note on the evolution of a surface by its mean curvature, Duke Math. J. 58, 555-558.

[22] G. Huisken, (1984). Flow by mean curvature of convex surfaces into spheres, J. Differential Geom. 20, 237-266.

[23] Il'in, Kalaŝnikov and Olě̈nik, Second-order linear equations of parabolic type, (Russian) Uspehi Mat. Nauk 105(1962), 3-146; Tr. Semin. im. I. G. Petrovskogo (2001), 9-193, 341; translation in J. Math. Sci. 108(2002), 435542 .

[24] T. Ilmanen, (1993). Convergence of the Allen-Cahn equation to Brakke's motion by mean curvature, J. Differential. Geom. 38, 417-461.

[25] H. Kawarada, (1974/75). On solutions of initial-boundary value problem for $u_{t}=u_{x x}+1 /(1-u)$, Publ. Res. Inst. Math. Sci. 10, 729-736.

[26] A. A. Lacey, (1984). The form of blow-up for nonlinear parabolic equations, Proc. Roy. Soc. Edinburgh Sect. A 98, 183-202.

[27] O. A. Ladyzenskaja, V. A. Solonikov and N. N. Ural'ceva, 1968. Linear and Quasilinear Equations of Parabolic Type, Transl. Math. Monographs, 23, AMS Providence R. I.

[28] A. Lunardi, (1984). Abstract Quasilinear Parabolic Equations, Math. Ann. 267, 395-415.

[29] A. Lunardi, 1995. Analytic Semigroups and Optimal Regularity in Parabolic Problems, Birkhäuser, Basel.

[30] Y. Seki, (2008). On directional blow-up for quasilinear parabolic equations with fast diffusion, J. Math. Anal. Appl. 338, 572-587.

[31] Y. Seki, A remark on blow-up at space infinity, The proceedings for the seventh AIMS International Conference on Dynamical Systems, Differential Equations and applications 2008, submitted. 
[32] Y. Seki, R. Suzuki and N. Umeda, (2008). Blow-up directions for quasilinear parabolic equations, Proc. Roy. Soc. Edinburgh Sect. A 138, 379-405.

[33] M. Shimojō, (2008). The global profile of blow-up at space infinity for semilinear heat equations, J. Math. Kyoto Univ. 48, 339-361.

[34] H. M. Soner, (1993). Motion of a set by the curvature of its boundary, J. Differential Equations 101, 313-372.

[35] H. M. Soner and P. E. Souganidis, (1993). Singularities and uniqueness of cylindrically symmetric surfaces moving by mean curvature, Comm. Partial Differential Equations 18, 859-894.

[36] Y. Tonegawa, (2003). Integrality of varifolds in the singular limit of reaction-diffusion equations, Hiroshima Math. J. 33, 323-341.

[37] B. White, (1997). Stratification of minimal surfaces, mean curvature flows and harmonic maps, J. Reine. Angew. Math. 488, 1-35.

[38] B. White, (2003). The size of the singular set in mean curvature flow of mean convex sets, J. Amer. Math. Soc. 16, 123-138. 